\title{
Awareness of HPV Testing and Acceptability of Self-sampling for Cervical Cancer Screening Among Women in Minnesota
}

J Gen Intern Med 37(6):1565-8

DOI: $10.1007 / \mathrm{s} 11606-021-06854-x$

(c) The Author(s) 2021

\section{INTRODUCTION}

Recent US data reveal concerning declines in cervical cancer (CC) screening rates and persistent disparities in CC screening and related outcomes by sociodemographic factors. ${ }^{1,2}$ The U.S. Preventive Services Task Force endorsement of HPV testing as a primary approach to $\mathrm{CC}$ screening offers an opportunity to explore self-sampling (patient collection of a vaginal swab) as an alternative to clinician sampling. ${ }^{3}$ Selfsampled HPV testing with timely follow-up care has the potential to improve CC screening uptake particularly in low resource settings. With the goal to inform self-sampling interventions to improve HPV testing uptake among underscreened women, this study examines awareness and use of HPV testing and acceptability of self-sampling and differences by sociodemographic factors among CC screening-eligible women living in Minnesota.

\section{METHODS}

\section{Study Population}

Data were from the omnibus 2019 Minnesota State Survey implemented by the Minnesota Center for Survey Research at the University of Minnesota (UMN) through telephone interviews between October 2019 and March 2020, using a simple random sample of Minnesota adult residents with a landline or cell phone $(\mathrm{N}=612$; response rate $=10 \%)$. Telephone interviewing was abruptly terminated on March 16, 2020, when UMN suspended all on-campus work due to COVID-19. This study was approved by the UMN Institutional Review Board.

\section{Measures}

CC screening-eligible respondents (females ages 21-65 without hysterectomy; $\mathrm{N}=155$ ) self-reported whether they

Received February 9, 2021

Accepted April 22, 2021

Published online May 13, 2021 had heard of HPV testing for CC screening and whether they have had a HPV or Pap test. Respondents were also asked to compare self-sampling for HPV testing to Pap testing done by a clinician in terms of convenience, embarrassment, ease, and pain, and likelihood of following up abnormal results with further testing. Sociodemographic factors including race/ethnicity, education, marital status, household income, housing, and metropolitan area were measured.

\section{Statistical Analysis}

We report descriptive statistics for awareness of HPV testing as a screening option and CC screening history, and acceptance of self-sampling for HPV testing. We examined sociodemographic differences using chi-square test or Fisher's exact test.

\section{RESULTS}

Table 1 summarizes sample characteristics and HPV testing awareness and self-reported HPV and Pap testing history by sociodemographic factors. Among screening-eligible respondents, $64.5 \%$ reported they have heard of HPV testing and $34.7 \%$ reported they have had HPV testing, while $89.5 \%$ reported they have had Pap testing. Women ages 21-29 (versus 30-65) less frequently heard of HPV testing ( $p=.041)$ while women ages $21-29$ or 40 59 less frequently had HPV testing $(p=.002)$. Women without a college degree (versus college graduates) less frequently heard of or had HPV testing $(p=.014, .015)$. Additionally, women ages 21-29, racial/ethnic minority (versus non-Hispanic white) women, and women who rent (versus own) their homes less frequently had Pap testing ( $p=.044, .007, .015)$.

Table 2 summarizes acceptability of HPV testing self-sampling by sociodemographic factors. The majority of respondents reported self-sampling as more convenient (77.8\%), less embarrassing (68.6\%), easier (74.5\%), and less painful (62.7\%) compared to Pap testing done by a clinician. Women without a college degree more frequently rated self-sampling as less painful $(p<.001)$, while women living in the largely rural greater Minnesota region (versus Twin Cities metropolitan area) more 
Table 1 Awareness of HPV Testing Option and Patient-Reported Cervical Cancer Screening History by Sociodemographic Factors

\begin{tabular}{|c|c|c|c|c|c|c|c|}
\hline & \multirow[t]{2}{*}{$\begin{array}{l}\text { Total } \\
\text { N }(\%)\end{array}$} & \multicolumn{2}{|c|}{$\begin{array}{l}\text { Before today, have you } \\
\text { ever heard of using an } \\
\text { HPV test for cervical } \\
\text { cancer screening a } \\
(\mathrm{N}=155)\end{array}$} & \multicolumn{2}{|c|}{$\begin{array}{l}\text { Have you ever had an } \\
\text { HPV test to screen for } \\
\text { cervical cancer b } \\
(\mathrm{N}=150){ }^{c}\end{array}$} & \multicolumn{2}{|c|}{$\begin{array}{l}\text { Have you ever had a Pap } \\
\text { smear or Pap test to } \\
\text { screen for cervical } \\
\text { cancer } \\
(\mathrm{N}=153)\end{array}$} \\
\hline & & $\begin{array}{l}\text { N }(\%) \\
\text { Yes }\end{array}$ & $p$-value & $\begin{array}{l}\text { N }(\%) \\
\text { Yes }\end{array}$ & $p$-value & $\begin{array}{l}\text { N }(\%) \\
\text { Yes }\end{array}$ & $p$-value \\
\hline Total & 155 & $100(64.5)$ & & $52(34.7)$ & & $137(89.5)$ & \\
\hline $\begin{array}{l}\text { Age in years } \\
21-29 \\
30-39 \\
40-49 \\
50-59 \\
60-65\end{array}$ & $\begin{array}{ll}24 & (16.8) \\
36 & (25.2) \\
35 & (24.5) \\
32 & (22.4) \\
16 & (11.2)\end{array}$ & $\begin{array}{l}9(37.5) \\
26(72.2) \\
24(68.6) \\
18(56.2) \\
12(75.0)\end{array}$ & .041 & $\begin{array}{l}5(20.8) \\
18(51.4) \\
10(30.3) \\
4(12.9) \\
9(56.2)\end{array}$ & .002 & $\begin{array}{l}17(70.8) \\
34(94.4) \\
32(91.4) \\
29(90.6) \\
15(100)\end{array}$ & .044 \\
\hline $\begin{array}{l}\text { Race/ethnicity }{ }^{\mathrm{f}} \\
\text { Non-Hispanic white } \\
\text { Other race/ethnicity }\end{array}$ & $\begin{array}{l}121(80.7) \\
29(19.3)\end{array}$ & $\begin{array}{l}81(66.9) \\
15(51.7)\end{array}$ & .125 & $\begin{array}{l}42(35.6) \\
8(29.6)\end{array}$ & .556 & $\begin{array}{l}112(94.1) \\
22(75.9)\end{array}$ & .007 \\
\hline $\begin{array}{l}\text { Education } \\
\text { High school or lower } \\
\text { Some college } \\
\text { College graduate } \\
\text { Post graduate }\end{array}$ & $\begin{array}{l}23(14.9) \\
52(33.8) \\
43(27.9) \\
36(23.4)\end{array}$ & $\begin{array}{l}9(39.1) \\
32(61.5) \\
31(72.1) \\
28(77.8)\end{array}$ & .014 & $\begin{array}{l}3(13.0) \\
14(28.0) \\
18(42.9) \\
17(50.0)\end{array}$ & .015 & $\begin{array}{l}20(87.0) \\
46(88.5) \\
38(90.5) \\
32(91.4)\end{array}$ & .933 \\
\hline $\begin{array}{l}\text { Marital status } \\
\text { Married } \\
\text { Single } \\
\text { Separated/widowed/other }\end{array}$ & $\begin{array}{l}89(57.8) \\
44(28.6) \\
21(13.6)\end{array}$ & $\begin{array}{l}62(69.7) \\
26(59.1) \\
11(52.4)\end{array}$ & .231 & $\begin{array}{l}30(35.3) \\
16(36.4) \\
6(30.0)\end{array}$ & .879 & $\begin{array}{l}82(94.3) \\
37(84.1) \\
17(81.0)\end{array}$ & .052 \\
\hline $\begin{array}{l}\text { Household income }^{\mathrm{i}} \\
<\$ 30,000 \\
\$ 30,000-\$ 60,000 \\
\$ 60,000-\$ 90,000 \\
\$ 90,000-\$ 120,000 \\
\geq \$ 120,000\end{array}$ & $\begin{array}{l}22(16.9) \\
25(19.2) \\
31(23.8) \\
22(16.9) \\
30(23.1)\end{array}$ & $\begin{array}{l}8(36.4) \\
17(68.0) \\
21(67.7) \\
14(63.6) \\
21(70.0)\end{array}$ & .102 & $\begin{array}{l}2(9.1) \\
10(41.7) \\
13(41.9) \\
7(31.8) \\
10(35.7)\end{array}$ & .099 & $\begin{array}{l}18(81.8) \\
22(88.0) \\
26(86.7) \\
21(95.5) \\
29(96.7)\end{array}$ & .367 \\
\hline $\begin{array}{l}\text { Housing status } \\
\text { Own } \\
\text { Rent }\end{array}$ & $\begin{array}{l}112(72.7) \\
42(27.3)\end{array}$ & $\begin{array}{l}75(67.0) \\
24(57.1)\end{array}$ & .257 & $\begin{array}{l}37(34.3) \\
14(34.1)\end{array}$ & .990 & $\begin{array}{l}103(93.6) \\
33(78.6)\end{array}$ & .015 \\
\hline $\begin{array}{l}\text { Metropolitan area } \\
\text { Greater Minnesota } \\
\text { Twin Cities area }\end{array}$ & $\begin{array}{l}60(38.7) \\
95(61.3)\end{array}$ & $\begin{array}{l}44(73.3) \\
56(58.9)\end{array}$ & .068 & $\begin{array}{l}18(30.5) \\
34(37.4)\end{array}$ & .389 & $\begin{array}{l}56(93.3) \\
81(87.1)\end{array}$ & .284 \\
\hline
\end{tabular}

p-values obtained from chi-square test or Fisher's exact test

${ }^{a}$ All participants were given a brief description of the HPV test before answering this question: "The Human Papillomavirus or HPV test is another method used for cervical cancer screening."

${ }^{b}$ Participants who answered "No" to the question "Before today, have you ever heard of using an HPV test for cervical cancer screening" did not receive this question and were coded as "No" for this question

${ }^{c}$ Missing response $=5$

${ }^{d}$ Missing response $=1$

${ }^{e}$ Missing response $=12$

${ }^{f}$ Missing response $=5$

${ }^{g}$ Missing response $=1$

${ }^{h}$ Missing response $=1$

${ }^{i}$ Missing response $=25$

${ }^{j}$ Missing response $=1$

frequently rated self-sampling as more convenient and easier $(p=.041, .002)$. Lastly, $94.8 \%$ of respondents reported that they would be likely to follow up with further testing upon receiving an abnormal result from the self-sampled HPV test.

\section{DISCUSSION}

Self-sampling for HPV testing was perceived as more convenient, less embarrassing, easier, and less painful than clinician sampling for Pap testing by most screening-eligible women we surveyed, especially women without a college degree and 
Table 2 Acceptability of Self-sampling for HPV Testing by Sociodemographic Factors

\begin{tabular}{|c|c|c|c|c|c|c|c|c|c|c|}
\hline & \multicolumn{8}{|c|}{$\begin{array}{l}\text { Compared to having a Pap test done by your health care provider, collecting your own } \\
\text { vaginal sample at home would be... a }\end{array}$} & \multirow{2}{*}{\multicolumn{2}{|c|}{$\begin{array}{l}\text { How likely would } \\
\text { you be to follow up } \\
\text { with your health } \\
\text { care provider for } \\
\text { further testing if } \\
\text { you received an } \\
\text { abnormal result } \\
\text { from your HPV } \\
\text { self-sampling f } \\
(\mathrm{N}=154)\end{array}$}} \\
\hline & \multicolumn{2}{|c|}{$\begin{array}{l}\text { More convenient } \\
(\mathbf{N}=\mathbf{1 5 3})^{b}\end{array}$} & \multicolumn{2}{|c|}{$\begin{array}{l}\text { Less embarrassing } \\
(\mathrm{N}=153)\end{array}$} & \multicolumn{2}{|l|}{$\begin{array}{l}\text { Easier } \\
(\mathbf{N}=153)\end{array}$} & \multicolumn{2}{|c|}{$\begin{array}{l}\text { Less painful } \\
(\mathrm{N}=134)\end{array}$} & & \\
\hline & $\begin{array}{l}\text { N }(\%) \\
\text { Agree }\end{array}$ & $\begin{array}{l}p \text { - } \\
\text { value }\end{array}$ & $\begin{array}{l}\text { N }(\%) \\
\text { Agree }\end{array}$ & $\begin{array}{l}p \text { - } \\
\text { value }\end{array}$ & $\begin{array}{l}\text { N (\%) } \\
\text { Agree }\end{array}$ & $\begin{array}{l}p \text { - } \\
\text { value }\end{array}$ & $\begin{array}{l}\text { N (\%) } \\
\text { Agree }\end{array}$ & $\begin{array}{l}p \text { - } \\
\text { value }\end{array}$ & $\begin{array}{l}\text { N }(\%) \\
\text { Likely }\end{array}$ & $\begin{array}{l}p \text { - } \\
\text { value }\end{array}$ \\
\hline Total & $119(77.8)$ & & 105 (68.6) & & $114(74.5)$ & & $84(62.7)$ & & $146(94.8)$ & \\
\hline $\begin{array}{l}\text { Age in years } \\
21-29 \\
30-39 \\
40-49 \\
50-59 \\
60-65\end{array}$ & $\begin{array}{l}21(91.3) \\
28(77.8) \\
24(70.6) \\
26(81.2) \\
12(75.0)\end{array}$ & .423 & $\begin{array}{l}17(77.3) \\
23(63.9) \\
26(74.3) \\
24(75.0) \\
11(68.8)\end{array}$ & .791 & $\begin{array}{l}20(87.0) \\
26(72.2) \\
27(79.4) \\
23(71.9) \\
12(75.0)\end{array}$ & .675 & $\begin{array}{l}15(68.2) \\
17(56.7) \\
19(63.3) \\
17(60.7) \\
12(80.0)\end{array}$ & .614 & $\begin{array}{l}24(100) \\
32(88.9) \\
34(97.1) \\
31(96.9) \\
16(100)\end{array}$ & .309 \\
\hline $\begin{array}{l}\text { Race/ethnicity }{ }^{\mathrm{i}} \\
\text { Non-Hispanic white } \\
\text { Other race/ethnicity }\end{array}$ & $\begin{array}{l}97(80.8) \\
20(71.4)\end{array}$ & .271 & $\begin{array}{l}88(72.7) \\
16(59.3)\end{array}$ & .166 & $\begin{array}{l}90(75.0) \\
22(78.6)\end{array}$ & .692 & $\begin{array}{l}63(61.2) \\
20(71.4)\end{array}$ & .318 & $\begin{array}{l}116(95.9) \\
27(93.1)\end{array}$ & .621 \\
\hline $\begin{array}{l}\text { Education }{ }^{\mathrm{j}} \\
\text { High school or lower } \\
\text { Some college } \\
\text { College graduate } \\
\text { Post graduate }\end{array}$ & $\begin{array}{l}20(87.0) \\
40(76.9) \\
30(71.4) \\
28(80.0)\end{array}$ & .530 & $\begin{array}{l}19(82.6) \\
39(76.5) \\
23(54.8) \\
24(66.7)\end{array}$ & .060 & $\begin{array}{l}20(87.0) \\
43(82.7) \\
27(64.3) \\
24(68.6)\end{array}$ & .081 & $\begin{array}{l}17(81.0) \\
37(78.7) \\
13(36.1) \\
17(58.6)\end{array}$ & $<.001$ & $\begin{array}{l}21(95.5) \\
49(94.2) \\
42(97.7) \\
34(94.4)\end{array}$ & .904 \\
\hline $\begin{array}{l}\text { Marital status } \\
\text { Married } \\
\text { Single } \\
\text { Separated/widowed/other }\end{array}$ & $\begin{array}{l}67(76.1) \\
34(79.1) \\
18(85.7)\end{array}$ & .726 & $\begin{array}{l}59(66.3) \\
28(66.7) \\
18(85.7)\end{array}$ & .206 & $\begin{array}{l}63(71.6) \\
33(76.7) \\
18(85.7)\end{array}$ & .387 & $\begin{array}{l}49(62.0) \\
23(60.5) \\
12(70.6)\end{array}$ & .762 & $\begin{array}{l}86(96.6) \\
41(95.3) \\
19(90.5)\end{array}$ & .327 \\
\hline $\begin{array}{l}\text { Household Income } \\
<\$ 30,000 \\
\$ 30,000-\$ 60,000 \\
\$ 60,000-\$ 90,000 \\
\$ 90,000-\$ 120,000 \\
\geq \$ 120,000\end{array}$ & $\begin{array}{l}17(77.3) \\
18(72.0) \\
25(83.3) \\
19(86.4) \\
23(79.3)\end{array}$ & .775 & $\begin{array}{l}15(68.2) \\
18(75.0) \\
23(76.7) \\
20(90.9) \\
17(56.7)\end{array}$ & .089 & $\begin{array}{l}17(77.3) \\
20(80.0) \\
22(75.9) \\
21(95.5) \\
19(63.3)\end{array}$ & .090 & $\begin{array}{l}16(72.7) \\
18(78.3) \\
17(70.8) \\
11(55.0) \\
12(48.0)\end{array}$ & .149 & $\begin{array}{l}19(86.4) \\
25(100) \\
28(90.3) \\
21(95.5) \\
30(100)\end{array}$ & .082 \\
\hline $\begin{array}{l}\text { Housing status }{ }^{m} \\
\text { Own } \\
\text { Rent }\end{array}$ & $\begin{array}{l}90(81.1) \\
29(70.7)\end{array}$ & .170 & $\begin{array}{l}79(70.5) \\
26(65.0)\end{array}$ & .516 & $\begin{array}{l}84(75.7) \\
30(73.2)\end{array}$ & .752 & $\begin{array}{l}58(62.4) \\
26(65.0)\end{array}$ & .773 & $\begin{array}{l}106(95.5) \\
39(92.9)\end{array}$ & .685 \\
\hline $\begin{array}{l}\text { Metropolitan area } \\
\text { Greater Minnesota } \\
\text { Twin Cities area }\end{array}$ & $\begin{array}{l}51(86.4) \\
68(72.3)\end{array}$ & .041 & $\begin{array}{l}45(75.0) \\
60(64.5)\end{array}$ & 172 & $\begin{array}{l}52(88.1) \\
62(66.0)\end{array}$ & .002 & $\begin{array}{l}38(69.1) \\
46(58.2)\end{array}$ & .201 & $\begin{array}{l}55(91.7) \\
91(96.8)\end{array}$ & .263 \\
\hline $\begin{array}{l}{ }^{a} \text { Respondents were given a } \\
\text { can be done by women in t } \\
\text { were measured on a 4-poin } \\
\text { two categories: somewhat } \\
{ }^{b} \text { Missing response }=2 \\
{ }^{c} \text { Missing response }=2 \\
{ }^{d} \text { Missing response }=2 \\
{ }^{e} \text { Missing response }=21 \\
{ }^{f} \text { Question was measured on } \\
\text { categories: } \text { somewhat to ve } \\
{ }^{g} \text { Missing response }=1 \\
{ }^{h} \text { Missing response }=12 \\
{ }^{i} \text { Missing response }=5 \\
{ }^{j} \text { Missing response }=1 \\
{ }_{k} \text { Missing response }=1 \\
{ }^{l} \text { Missing response }=25 \\
{ }_{m} \text { Missing response }=1\end{array}$ & $\begin{array}{l}\text { ief descrip } \\
\text { homes th } \\
\text { ikert style } \\
\text { 4-point Lil } \\
\text { likely and }\end{array}$ & $\begin{array}{l}\text { of } H \\
\text { gh sel } \\
\text { ale: st } \\
\text { and }\end{array}$ & $\begin{array}{l}\text { ct test } \\
\text { st self-sam } \\
\text { pling wher } \\
\text { y agree, sc } \\
\text { what to str } \\
\text { very likely } \\
\text { ery unlike }\end{array}$ & $\begin{array}{l}\text { hg bef } \\
\text { hey ar } \\
\text { what } \\
\text { ly dis }\end{array}$ & $\begin{array}{l}\text { swering } \\
\text { ided a kit } \\
\text { somewh }\end{array}$ & $\begin{array}{l}\text { que } \\
\text { a s } \\
\text { sagr }\end{array}$ & $\begin{array}{l}\text { "The H } \\
\text { collect t } \\
\text { ongly dis }\end{array}$ & est tc & $\begin{array}{l}\text { en for cerv } \\
\text { al sample. } \\
\text { ses were gi }\end{array}$ & $\begin{array}{l}l \text { cancer } \\
\text { uestions } \\
\text { ped into }\end{array}$ \\
\hline
\end{tabular}


women living in the greater Minnesota region. Additionally, over $90 \%$ of women reported they would seek follow-up testing of an abnormal result from a self-sampled HPV test. These findings suggest that self-sampling has the potential to improve HPV testing uptake for women in medically underserved and rural communities. The disparities by age, education, race/ethnicity, and housing in HPV testing awareness and reported $\mathrm{CC}$ screening history confirm previous findings, 4,5 suggesting interventions promoting HPV testing and selfsampling should focus on underserved populations to reduce existing CC disparities. These data can inform interventions to improve HPV testing uptake among underscreened women through self-sampling.

Limitations of this research include the low response rate, which may introduce bias and limit the generalizability, and the small sample size, which limited statistical power for exploring interaction effects.

Xuan $\mathrm{Zhu}, \mathrm{Ph} \mathrm{D}^{1}$

Kathy L. MacLaughlin, $M D^{2}$

Chun Fan, $B^{3}$

Debra J. Jacobson, $M S^{3}$

Gregory D. Jenkins, $\mathrm{MS}^{3}$

Robert M. Jacobson, $M D^{1,4}$

Lila J. Finney Rutten, $\mathrm{PhD}, \mathrm{MPH}^{1,5}$

${ }^{1}$ Robert D. and Patricia E. Kern Center for the Science of Health Care Delivery, Mayo Clinic,

Rochester, MN, USA

${ }^{2}$ Department of Family Medicine, Mayo Clinic,

Rochester, MN, USA

${ }^{3}$ Department of Quantitative Health Sciences, Mayo Clinic,

Rochester, MN, USA

${ }^{4}$ Department of Pediatric and Adolescent Medicine, Mayo Clinic, Rochester,

Rochester, MN, USA

${ }^{5}$ Division of Epidemiology, Mayo Clinic, Rochester, MN, USA
Corresponding Author: Xuan Zhu, PhD; Robert D. and Patricia E. Kern Center for the Science of Health Care Delivery, Mayo Clinic, Rochester, MN, USA (e-mail: zhu.xuan@mayo.edu).

\section{Declarations:}

Conflict of Interest: R.M.J. serves as a member of a safety review committee for post-licensure studies of human papillomavirus vaccines conducted by Merck and Co. and as a member of a data monitoring committee for a set of prelicensure trials of a 15-valent pneumococcal conjugate vaccine also conducted by Merck and Co.

Open Access This article is licensed under a Creative Commons Attribution 4.0 International License, which permits use, sharing, adaptation, distribution and reproduction in any medium or format, as long as you give appropriate credit to the original author(s) and the source, provide a link to the Creative Commons licence, and indicate if changes were made. The images or other third party material in this article are included in the article's Creative Commons licence, unless indicated otherwise in a credit line to the material. If material is not included in the article's Creative Commons licence and your intended use is not permitted by statutory regulation or exceeds the permitted use, you will need to obtain permission directly from the copyright holder. To view a copy of this licence, visit http://creativecommons. org/licenses/by/4.0/.

\section{REFERENCES}

1. Watson M, Benard V, King J, Crawford A, Saraiya M. National assessment of HPV and Pap tests: Changes in cervical cancer screening, National Health Interview Survey, Prev Med. 2017;100: 243-247.

2. National Cancer Institute. Cancer Trends Progress Report. Available from URL: https://progressreport.cancer.gov/detection/cervical_cancer. Accessed 9 May 2020

3. USPSTF Task Force, Curry SJ, Krist AH, Owens DK, Barry MJ, Caughey AB, et al. Screening for Cervical Cancer: US Preventive Services Task Force Recommendation Statement. JAMA. 2018;320: 674-686.

4. Hall IJ, Tangka FKL, Sabatino SA, Thompson TD, Graubard BI, Breen N. Patterns and Trends in Cancer Screening in the United States. Prev Chron Dis. 2018;15: E97-E97.

5. Johnson NL, Head KJ, Scott SF, Zimet GD. Persistent Disparities in Cervical Cancer Screening Uptake: Knowledge and Sociodemographic Determinants of Papanicolaou and Human Papillomavirus Testing Among Women in the United States. Publ Health Rep. 2020;135: 483-491.

Publisher's Note: Springer Nature remains neutral with regard to jurisdictional claims in published maps and institutional affiliations. 